\title{
New results on angular reconstruction of deep pulser radio signals
}

\section{Geoffrey Gaswint* for the ARIANNA collaboration ${ }^{\dagger}$}

University of California, Irvine, USA E-mail: ggaswint @uci . edu

\begin{abstract}
High energy neutrino detection via radio emission from the interaction vertex is an efficient method for neutrino energies between $10^{16} \mathrm{eV}$ and $10^{20} \mathrm{eV}$. The ARIANNA radio "Askaryan" detectors are located in the Antarctic ice just beneath the surface. Neutrino observation requires that radio pulses propagate to the surface with minimum distortion by the ice and firn medium. Using the residual hole from the South Pole Ice Core Project, radio pulses were sent at $1 \mathrm{~Hz}$ from a transmitter located up to $1.7 \mathrm{~km}$ below the snow surface. We quantify the direction reconstruction abilities of ARIANNA and refractive bending of signal trajectories in the firn using radio pulse data from 2018. After deconvolving the raw signals for amplifier and antenna response and correcting for cable delays, the electric fields reveal no significant distortion, e.g. birefringence, due to ice propagation. These studies also suggest that the polarization of the electric field, which is required to measure the direction of the neutrino, is not altered by the natural ice medium to a significant extent.
\end{abstract}

36th International Cosmic Ray Conference -ICRC2019-

July 24th - August 1st, 2019

Madison, WI, U.S.A.

\footnotetext{
${ }^{*}$ Speaker.

${ }^{\dagger}$ for collaboration list see PoS(ICRC2019)1177
} 


\section{Introduction}

ARIANNA detects neutrinos through radio Askaryan radiation created when ultra-high energy neutrinos interact with the ice. Ice serves as an excellent medium for radio propagation with attenuation lengths on the order of a kilometer. Using the large sheets of ice found in (Ant)artic regions and ARIANNA's surface detector design, a cost efficient array of neutrino detectors can be constructed to probe ultra-high energy neutrinos. ARIANNA currently has a pilot array in Moore's Bay, Antarctica with 8 stations plus 2 more located at the South Pole. The direction of high energy neutrinos requires both an accurate measurement of the incoming direction and polarization of the radio pulse generated from the neutrino interaction in the ice.

The reconstruction accuracy of the ARIANNA station has been studied previously. Surface pulsers directed radio pulses toward the bottom water-ice interface at Moore's Bay. The absolute measurement of the arrival direction of the reflected signal agreed with expectation to within 1 deg or better [1]. Reflected signals also demonstrated that polarization of the electric field was preserved during propagation and reflection [2]. Though these studies were encouraging, they were mostly confined to nearly vertical propagation.

Cosmic ray interactions in the atmosphere generate radio pulses that act as a calibration source. The ARIANNA stations configured with upward facing antennas accurately reconstruct the polarization and direction of incoming radio pulses over a much broader range of incoming angles and physical conditions (see also [3, 4])

To further test the reconstruction abilities of ARIANNA for radio signals that propagate through the ice medium, a radio pulser was lowered in a fluid-filled hole provided by the South Pole Ice Coring Experiment (SPICE) to a depth of $1.7 \mathrm{~km}$. We describe the preliminary results from these studies.

\section{Measurement Setup}

For this proceeding, we will focus on data collected in December 2018 by ARIANNA station 51 located about $1 \mathrm{~km}$ from South Pole Station and $0.65 \mathrm{~km}$ from the SPICE hole. [5]. The transmitter, developed by University of Kansas, broadcast short duration radio frequency pulses with a repetition rate of $1 \mathrm{~Hz}$. The vertically-oriented transmitter, containing a bicone antenna, was lowered to a depth of $1.7 \mathrm{~km}$. It is plausible that the SPICE core may be tilted by $1^{\circ}$.

ARIANNA station 51 consists of 8 antennas - 4 down facing LPDAs oriented in a square pattern with $6 \mathrm{~m}$ sides, and 4 vertically-oriented dipoles located at the corners of the square. The LPDAs provide two orthogonal Hpol (parallel to the surface) measurements and the dipoles measure the Vpol component. Several hundred events were transferred over the Iridium satellite Network for offline analysis.

\section{Ray Tracing Solution}

The density, and therefore the index-of-refraction, changes in the upper $200 \mathrm{~m}$ of the South Pole ice sheet from $n=1.78$ of deep ice to about $n=1.35$ at the surface. As a consequence, radio signals do not propagate rectalinearly but are refracted as illustrated in Fig. 1. Gray shading 
indicates the range of depth of the transmitter that permit no classical propagation solutions, which is termed the shadow zone. At greater depth, standard refraction propagation is assumed. The ray tracer of NuRadioMC [6] was used to compute the expected arrival direction, based on the "SP1" index-of-refraction profile that was derived from density measurements [7]. Two representative solutions are shown for the pulser at a depth of $1 \mathrm{~km}$ and $1.7 \mathrm{~km}$. The right panel gives the expected zenith angle at the ARIANNA station as a function of transmitter depth.
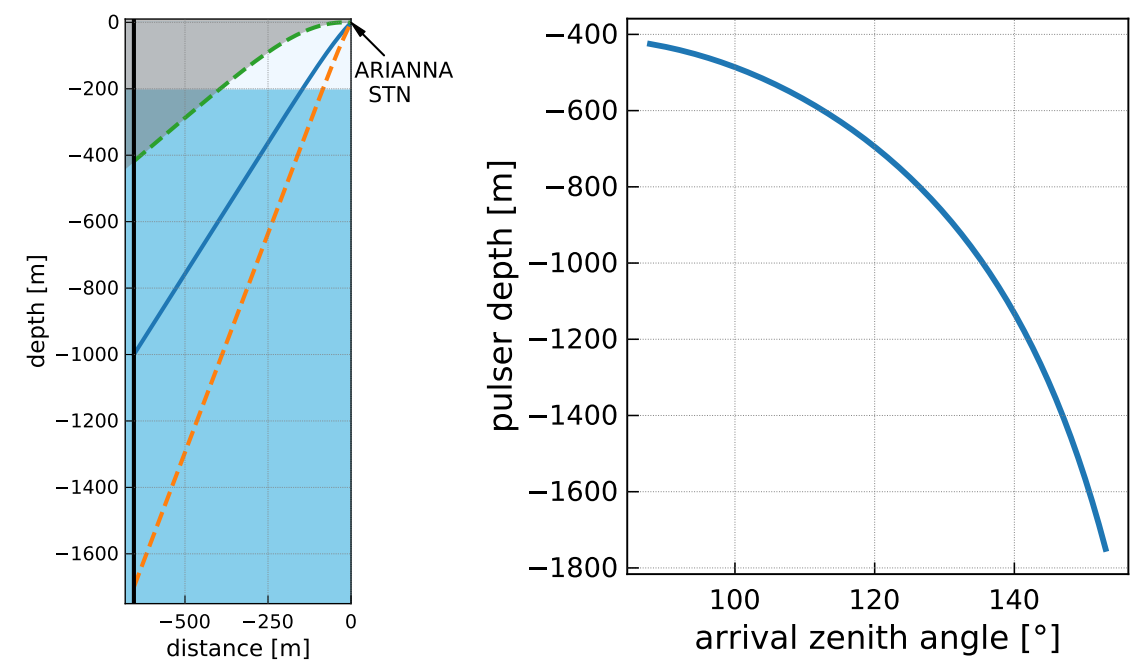

Figure 1: (left) Ray tracing solution for a transmitter at depths $418 \mathrm{~m}, 1 \mathrm{~km}$ and $1.7 \mathrm{~km}$ to the South Pole ARIANNA station 51 calculated with the NuRadioMC package. Light blue shaded region above $200 \mathrm{~m}$ is the firn layer. The grayed out area is the shadow zone. Vertical black line on the y axis represents the SPICE core. (Right) Expected arrival zenith angle versus transmitter depth.

\section{Direction Reconstruction}

The framework NuRadioReco [8] is used to reconstruct the incoming direction of a triggered event in the ARIANNA detector. The particular algorithm used is called the cross correlation method as it uses the time differences between two parallel pairs of antennas (found through correlating the two signals together) to determine the signal arrival direction. See [8] for details of the reconstruction algorithm.

A typical raw event from the deep pulser run in the SPICE hole is shown in Fig. 2. The transmitter was at a depth of $1 \mathrm{~km}$ during this event. The first four channels (0-3) are the four down facing LPDAs while the last four channels (4-7) are the vertically-orientated dipoles. The differences between the traces are associated with the different antenna types, amp variations, and cable lengths.

Before computing the arrival direction of each event, a few prepossessing steps are performed. First, The ARIANNA station saturates when the signal amplitude exceeds $600 \mathrm{mV}$. During this SPICE core run, events in the linear regime occur at depths below $800 \mathrm{~m}$ and only these events are considered in the following. After this a butterworth band pass filter of order 10 with a passband of $120 \mathrm{MHz}$ to $300 \mathrm{MHz}$ and zero group delay is applied to the traces followed by a rectangular bandpass filter between $10 \mathrm{MHz}$ and $1 \mathrm{GHz}$ to remove any noise from outside the sensitive bandwidth. 


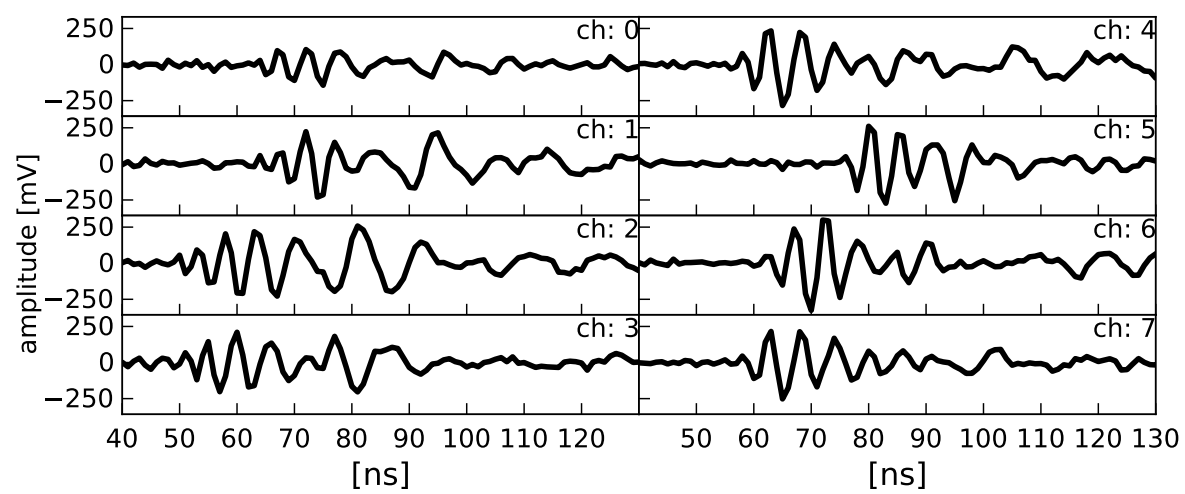

Figure 2: Raw triggered event in South Pole ARIANNA station 51. Channels 0-3 correspond to LPDAs, while channels 4-7 correspond to dipoles. Transmitter was located at $1 \mathrm{~km}$ depth.

The incoming radio signal has a frequency spectrum peaking around $250 \mathrm{MHz}$ so these filters aid in removing unwanted noise in our trace. After the filtering, the amplifier response is deconvolved. The approximate time delays from cable and electronics (as measured in the lab) are subtracted, but the accuracy was only $0.5 \mathrm{~ns}$. The next paragraph describes the procedure to improve the accuracy of the time delays. The traces are then up-sampled from $1 \mathrm{GHz}$ to $50 \mathrm{GHz}$ to improve the timing resolution. As we are only interested in the timing of the main signal pulse, a hanning window with a width of $50 \mathrm{~ns}$ around the pulse maximum is applied in the time domain to remove afterpulses and other artifacts.

Systematic errors in the lab values for the time offsets was uncovered by noting that the errors did not depend on depth. Therefore, we adjust the value for the time delay by a quantity $\Delta \mathrm{T}$, which is computed from expected time delays between each channel due to their spatial separation according to the ray tracing solutions found from NuRadioMC and then subtract the residual time delays of the signal pulse against one reference channel (channel 3 for the LPDAs and channel 6 for the dipoles). The resulting distribution of $\Delta \mathrm{T}$ is presented in Fig. 3. We observe offsets of up to $1 \mathrm{~ns}$ between channels with a standard deviation of $\sim 0.15 \mathrm{~ns}$. Since the variation is much smaller than the mean offset, the non-zero value is assumed to originate from cable delays or other delays along the signal chain. The depth dependence will be studied further at the end of this section.

Due to the common antenna correction, we chose to measure the arrival direction using the four LPDA waveforms only. A second independent measurement was constructed from the four dipole antennas. The signal arrival direction was reconstructed after correcting for the constant time offsets between channels found in Fig. 3, and then subtracted by the expected arrival direction (see Fig. 4). The direction reconstruction is quite accurate and precise with deviations between $0.2^{\circ}$ to $0.3^{\circ}$ centered around $0^{\circ}$. It is important to have such a precise measurement for accurate reconstruction of the vertex direction using the DnR technique and neutrino direction reconstruction (see [3]).

Figure 5 shows the differences between the reconstructed arrival direction and the expected direction. We see remarkably good agreement in the zenith angle for both dipoles and LPDAs, though a slight depth dependence which may be due to a slight tilt of the SPICE hole, a slight error in the ice model, or some other systematic issue. The azimuth has a slight depth dependence in 

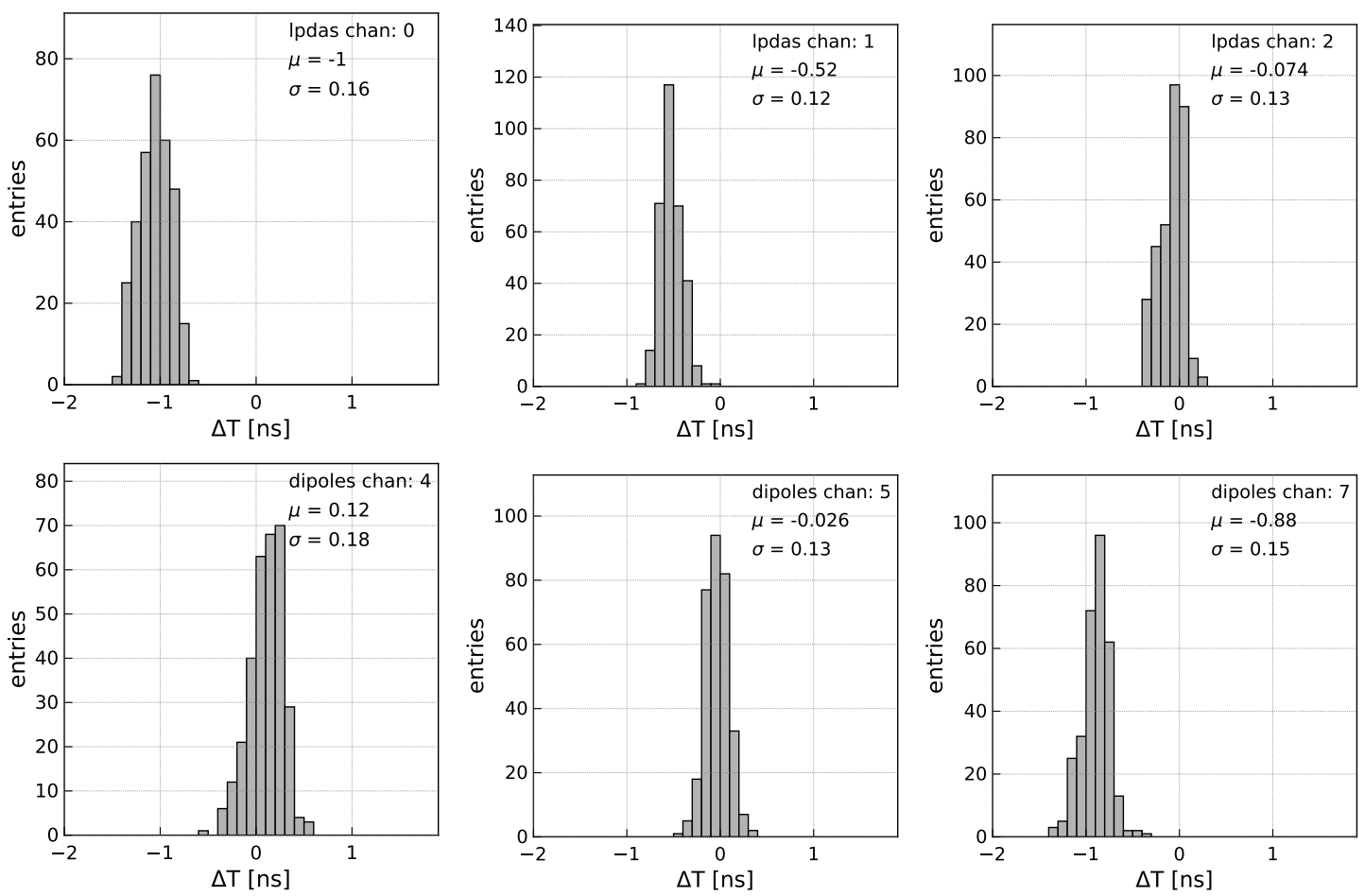

Figure 3: Time differences between channels after accounting for all the hardware and subtracting the expected time delays for each individual channel. First 4 histograms (top half) use channel 3 as the reference channel, whereas the last four histograms (bottom half) use channel 6 as a reference channel. These time delays can be associated with uncertainties in cable delays.
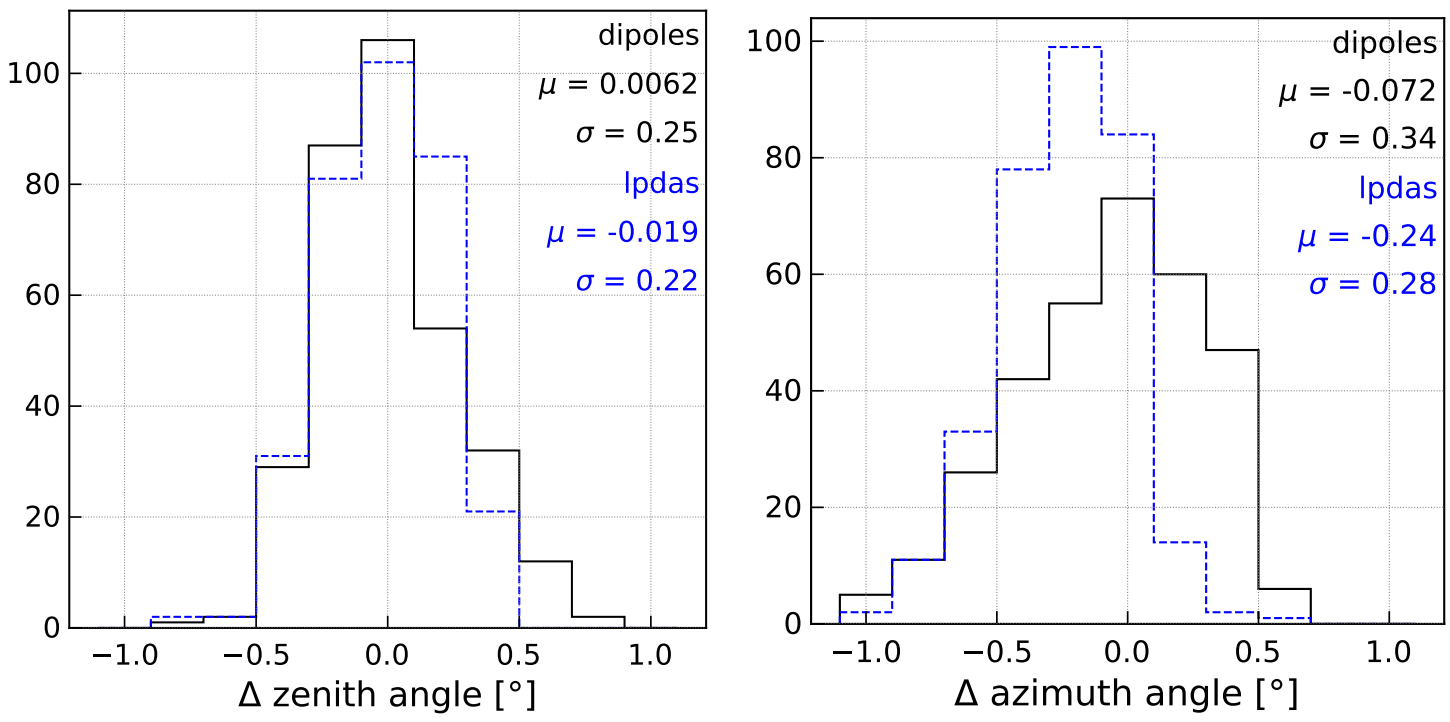

Figure 4: Reconstructed arrival direction minus expected arrival direction. Also factors in time differences between channels found in Fig. 3. Expected arrival direction is found using NuRadioMC ray tracer while the reconstructed direction uses the cross correlation method for finding the arrival direction. Blue curves show the residuals when only using the four LPDAs. Black curves show the residuals when only using the four dipoles. 
the dipoles. The LPDA results are more or less constant with depth. Ice cannot affect the azimuth direction since it would be orthogonal to the signal propagation. The dipoles azimuth and zenith vs depth trend similarly, providing evidence that the depth dependence is not an ice property. It rather suggests uncertainties in the dipole antenna positions.

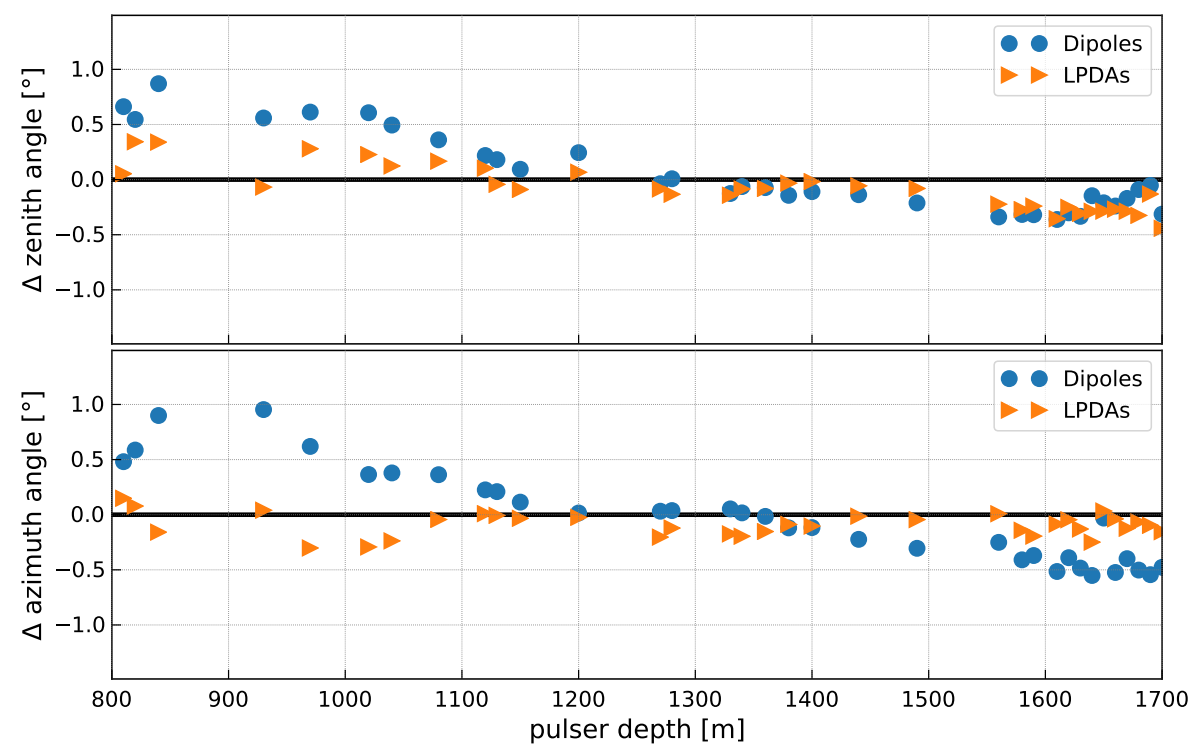

Figure 5: Scatter of the reconstructed angle minus the expected angle versus depth. This is the same data that went into making the residuals in figure 4

\section{Test of firn and ice effects}

The results from the previous section was used to test the impacts of the firn and ice on the propagation of radio signals. We present in Fig. 6 the measured pulses when the receiver was at $1 \mathrm{~km}$ and $1.7 \mathrm{~km}$ depth after the waveforms were corrected for the detector response. The antenna response was unfolded assuming a theta polarized signal, as the signal emitted from the dipole antennas is only theta polarized (vertical polarization for a horizontally going signal). The geometric time delays and the constant time offsets of Fig. 3 are factored in. The traces are upsampled to $50 \mathrm{GHz}$ to improve readability.

There is no evidence that the propagation through the firn and ice affects the radio signal. The shape of the signal pulses are almost identical between different channels and different transmitter depths, except for variations in the amplitudes, providing evidence that the signal is not significantly distorted during propagation through ice and firn. Furthermore, the observed pulse shape is compatible with the emitted signal pulse. We note that the channel-to-channel amplitude variation of the electric field is also constant with depth, likely due to a temperature dependence in the gain of the amplifiers. In addition, the electric fields recovered from the LDPAs show slightly smaller signal amplitudes than the electric fields recovered from the dipoles which points to systematic uncertainties in the antenna response pattern obtained from antenna simulations. Furthermore, boundary effects of the antenna being close to the ice surface are not considered in the antenna simulation used for this analysis. 
We also checked for net birefringence in the crystal orientation fabric by (1) searching for a time delay between the polarization parallel and perpendicular to the ice surface, and (2) searching for a consistent double pulse structure in commonly oriented antennas. The magnitude of the time delay is expected to increase with the propagation distance, i.e., the transmitter depth in our measurement. The waveforms in Fig 6 exhibit none of these features. We conclude that there is no evidence for significant birefringence in this data for the tested geometry where the velocity component parallel to the snow surface propagates in a direction roughly along the average ice flow direction.

Furthermore, we checked for a change of polarization during the signal propagation and find no evidence that the polarization is changed during propagation through the ice and firn: Due to the vertically-orientated transmitter, the polarization of the emitted electric field pulse is entirely theta polarized. Assuming that the polarization remains unchanged during propagation through the ice then when folding out the eight antennas in the ARIANNA station assuming an entirely theta polarized signal, the resulting electric fields in each channel should look identical. The dipole antennas are only sensitive to the theta component of the electric field (more precisely to the vertical projection of the theta component) whereas the LPDAs have a similar sensitivity to the theta and phi polarization of the electric field for the incoming signal directions studied here. Thus, the LPDA signal would get larger with respect to the dipoles the more phi-polarized the signal was. For a purely theta polarized signal, the electric fields presented in Fig. 6 should be equal. For any additional phi component of the signal, the electric fields of the LPDAs should become larger. As we recover a smaller electric field from the LPDA the most likely solution is a purely theta polarized signal as any additional phi component would further increase the tension. Thus, we find no indication in our data that the propagation through the firn/ice changes the polarization of the radio signal.

We are working on quantifying these effects and will report on it in an upcoming publication.

\section{Conclusions}

The angular reconstruction capabilities of the ARIANNA south pole station 51 is very precise with an error of $0.5^{\circ}$. There is no evidence that the firn distorts the waveform of radio signals. Further, there is no strong evidence for net birefringence effects. The polarization is theta polarized as expected for a vertically-orientated transmitter. More precise measurements of antenna locations and cable delays should be performed in the future. Future studies will include looking at shallower events (when the transmitter is in the shadow zone), and further development of the polarization reconstruction capabilities.

\section{Acknowledgements}

We are grateful to the U.S. National Science Foundation-Office of Polar Programs, the U.S. National Science Foundation-Physics Division (grant NSF-1607719) and the U.S. Department of Energy. We thank generous support from the German Research Foundation (DFG), grant NE 2031/2-1 and GL 914/1-1, the Taiwan Ministry of Science and Technology. H. Bernhoff acknowledge support from the Swedish Government strategic program Stand Up for Energy. E. Unger 

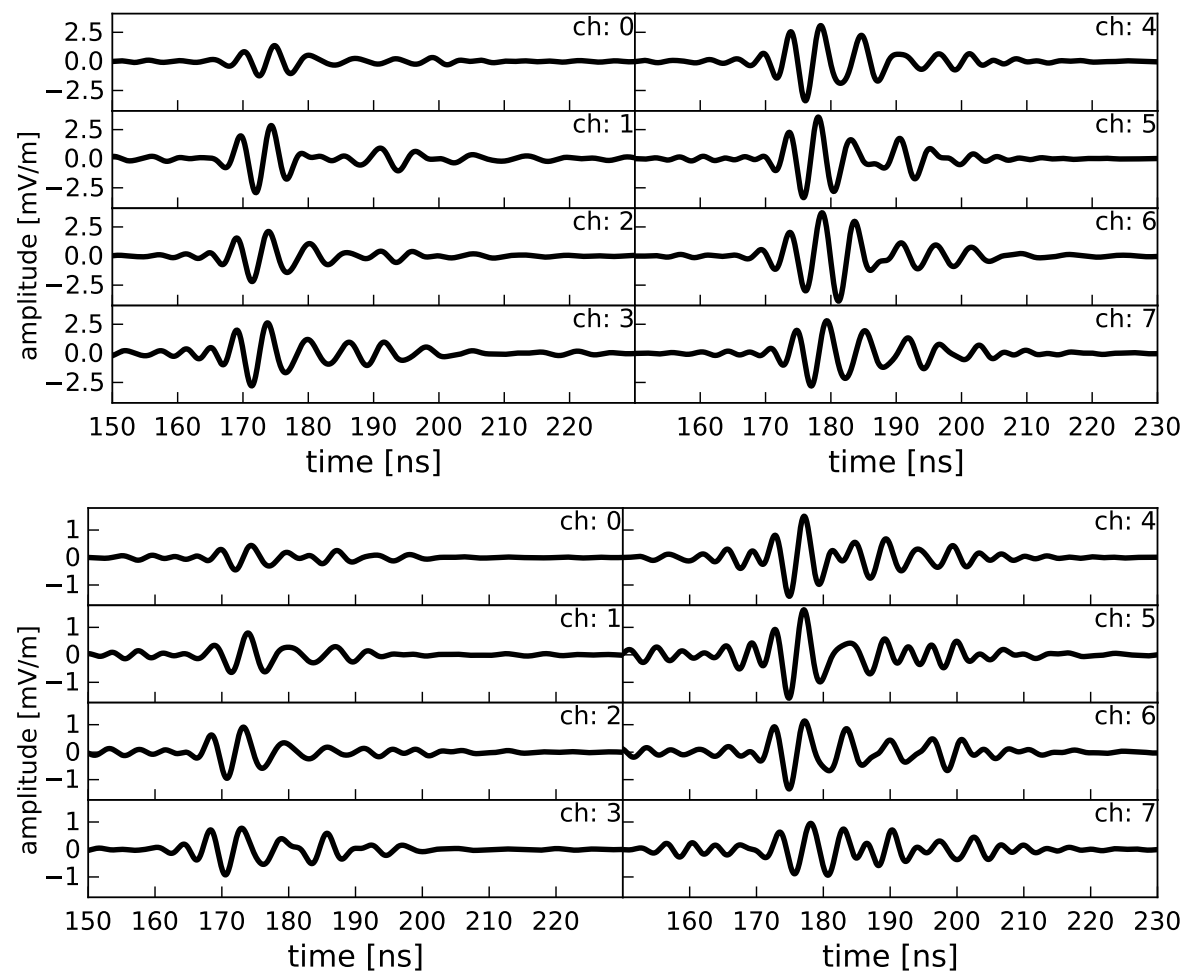

Figure 6: Electric field in each channel. Antenna response was deconvolved assuming the incoming electric field was entirely theta polarized. (Top) transmitter at $1 \mathrm{~km}$ depth, (bottom) transmitter at $1.7 \mathrm{~km}$ depth.

acknowledge support from the Uppsala university Vice-Chancellor's travel grant (sponsored by the Knut and Alice Wallenberg Foundation) and the C.F. Liljewalch travel scholarships. D. Besson and A. Novikov acknowledge support from the MEPhI Academic Excellence Project (Contract No. 02.a03.21.0005) and the Megagrant 2013 program of Russia, via agreement 14.12.31.0006 from 24.06.2013

\section{References}

[1] C. Reed for the ARIANNA collaboration, PoS(ICRC2015) (2015) [1509.00109].

[2] J. C. Hanson et al., Journal of Glaciology (2014) [1410 . 7134].

[3] C. Glaser for the ARIANNA collaboration, PoS(ICRC2019)899 (this proceedings) (2019) .

[4] A. Nelles for the ARIANNA collaboration, PoS(ICRC2019)366 (this proceedings) (2019) .

[5] A. Anker et al., Advances in Space Research (in press) (2019) [1903.01609].

[6] C. Glaser et al., submitted to EPJ-C (2019) [1906.01670].

[7] S. W. Barwick et al., JCAP07(2018)055 (2018) [1804.10430].

[8] C. Glaser et al., The European Physical Journal C 79 (2019) [1903.07023]. 